\title{
LOWER BOUNDS OF THE GAP BETWEEN THE FIRST AND SECOND EIGENVALUES OF THE SCHRÖDINGER OPERATOR
}

BY

QIHUANG YU AND JIA-QING ZHONG

\begin{abstract}
In this paper the authors prove the following theorem:
Let $\Omega$ be a smooth strictly convex bounded domain in $R^{n}$ and $V: \Omega \rightarrow R$ a nonnegative convex function. Suppose $\lambda_{1}$ and $\lambda_{2}$ are the first and second nonzero eigenvalues of the equation
\end{abstract}

$$
-\Delta f+V f=\lambda f,\left.\quad f\right|_{\partial \Omega} \equiv 0 .
$$

Then $\lambda_{2}-\lambda_{1} \geq \pi^{2} / d^{2}$, where $d$ is the diameter of $\Omega$.

Let $\Omega \subset R^{n}$ be a smooth strictly convex bounded domain and $W: \Omega \rightarrow R$ a nonnegative convex smooth function. The eigenvalues of the equation

$$
-\Delta f+W f=\lambda f, \quad f=0, \quad \text { on } \partial \Omega
$$

can be arranged in nondecreasing order as follows:

$$
0<\lambda_{1}<\lambda_{2} \leq \lambda_{3} \leq \cdots .
$$

B. Wong, S.-T. Yau and Stephen S.-T. Yau [3] proved that

$$
\lambda_{2}-\lambda_{1} \geq \pi^{2} / 4 d^{2}
$$

where $d$ is the diameter of $\Omega$. In this paper the authors will use the method of [3 and 4] to prove the following theorem:

THEOREM. Let $\Omega$ be a smooth strictly convex bounded domain in $R^{n}$ and $W: \Omega$ $\rightarrow R$ a nonnegative convex function. Suppose $\lambda_{1}$ and $\lambda_{2}$ are the first and second nonzero eigenvalues of (1). Then

$$
\lambda_{2}-\lambda_{1} \geq \pi^{2} / d^{2},
$$

where $d$ is the diameter of $\Omega$.

The authors are grateful to Professor S.-T. Yau for his kind direction and help, and to The Institute for Advanced Study, Princeton, N.J., for its support.

In this paper the assumptions of all the lemmas are the same as those of the Theorem. We will not state them again.

Let $f_{1}$ and $f_{2}$ be the first and second eigenfunctions of $(1)$; then $f_{1}(x)>0, x \in \Omega$ $[\mathbf{2}]$, and $u=f_{2} / f_{1}$ is smooth to the boundary of $\Omega[\mathbf{3}]$. Suppose that

$$
A=\max _{x \in \bar{\Omega}} u(x) ; \quad-k=\min _{x \in \bar{\Omega}} u(x) .
$$

Received by the editors April 24, 1985.

1980 Mathematics Subject Classification (1985 Revision). Primary 53C20. 
We may assume that $A \geq k$, otherwise, we can use $-f_{2}$ instead of $f_{2}$.

Since $\int_{\Omega} f_{1} f_{2}=0$ and $f_{1}>0, k>0$. Setting $\tilde{u}=u / A$, we have $1 \geq \tilde{u} \geq-k / A=$ $-\tilde{k}$ and $1 \geq \tilde{k}>0$

$$
v=\left(\tilde{u}-\frac{1-\tilde{k}}{2}\right) /\left(\frac{1+\tilde{k}}{2}\right)
$$

and

$$
a=\frac{1-\tilde{k}}{1+\tilde{k}} ; \quad 1>a \geq 0 .
$$

Then $v$ is a smooth function on $\bar{\Omega}$. By computing, we have

$$
\Delta v=-\lambda(v+a)-2\left(\nabla v \cdot \nabla \log f_{1}\right),
$$

where $\lambda$ is $\lambda_{2}-\lambda_{1}>0$.

LEMMA 1. Let $z(v)$ be a smooth function defined on $\bar{\Omega}$ and $m>0$ a constant. Suppose that

$$
G(x)=m|\nabla v|^{2} \neq z(v)
$$

$P \in \partial \Omega$ and $G(P)=\max _{x \in \bar{\Omega}} G(x)$. Then $\nabla v(P)=0$.

PROOF. We can choose an orthonormal frame $l_{1}, l_{2}, \ldots, l_{n}$ around $P$ such that $l_{1}$ is perpendicular to $\partial \Omega$ and pointing outward. We also use the notation $\partial / \partial x_{1}$ to denote the restriction of $l_{1}$ on $\partial \Omega$. Since $G(P)$ is the maximum of $G(x)$,

$$
0 \leq \frac{\partial G}{\partial x_{1}}(P)=2 m \sum_{i=1}^{n} v_{i} v_{i 1}+z^{\prime} v_{1} .
$$

Furthermore, in $\Delta v=-\lambda(v+a)-2\left(\nabla v \cdot \nabla \log f_{1}\right), v$ and $\Delta v$ are all smooth on $\bar{\Omega}$; hence

$$
\nabla v \cdot \nabla \log f_{1}=\frac{1}{f_{1}}\left[v_{1}\left(f_{1}\right)_{1}+\sum_{i=2}^{n} v_{i}\left(f_{1}\right)_{i}\right]
$$

achieves finite value on $\partial \Omega$. But $\left.f_{1}\right|_{\partial \Omega} \equiv 0$, thus

$$
\left.\left[v_{1}\left(f_{1}\right)_{1}+\sum_{i=2}^{n} v_{i}\left(f_{1}\right)_{i}\right]\right|_{\partial \Omega} \equiv 0 .
$$

Since $f_{1} \equiv 0$ and $\partial \Omega$ and $l_{i}, 2 \leq i \leq n$, are the tangent vectors of $\partial \Omega,\left.\left(f_{1}\right)_{i}\right|_{\partial \Omega} \equiv 0$, $2 \leq i \leq n$. Hence,

$$
v_{1}\left(f_{1}\right)_{1} \equiv 0 \quad \text { on } \partial \Omega
$$

By Hopf's lemma, $\partial f_{1} / \partial x_{1} \neq 0$. Therefore,

$$
v_{1} \equiv 0 \quad \text { on } \partial \Omega \text {. }
$$

Putting (9) into (8), we have

$$
0 \leq m \sum_{i=2}^{n} v_{i} v_{i 1}(P)=\frac{\partial G}{\partial x_{1}}(P) .
$$


From the definition of the second fundamental form in $R^{n}$, we have (note $v_{1}=0$ )

$$
v_{1 i}=-\sum_{j=2}^{n} h_{i j} v_{j},
$$

where $\left(h_{i j}\right)$ is the second fundamental form. Putting (11) into (10), we obtain

$$
0 \leq-\sum_{i, j=2}^{n} m h_{i j} v_{i} v_{j}(P) .
$$

Since $\Omega$ is strictly convex, $\left(h_{i j}\right)$ is positive defifnite; thus

$$
0 \leq-m \sum_{i, j=2}^{n} h_{i j} v_{i} v_{j}(P) \leq 0 .
$$

Hence, $v_{i}(P)=0,2 \leq i \leq n$, i.e., $\nabla v(P)=0$.

LEMMA 2. For any given $b>1$,

$$
\frac{|\nabla v|^{2}}{b^{2}-v^{2}} \leq \lambda(1+a)
$$

PROOF. For $\varepsilon>0$, consider the function defined on $\bar{\Omega}$

$$
G(x)=|\nabla v|^{2}+\lambda(1+\varepsilon+a) v^{2} .
$$

Suppose $G(P)=\max _{x \in \bar{\Omega}} G(x)$. If $P \in \partial \Omega$, by Lemma 1 , we have $\nabla v(P)=0$ and

$$
G(x) \leq G(P)=\lambda(1+\varepsilon+a) v^{2}(P) \leq \lambda(1+\varepsilon+a) .
$$

Now we assume that $P \in \Omega$, and from the maximum principle we have that at $P$

$$
0=\nabla|\nabla v|^{2}+\lambda(1+\varepsilon+a) \nabla v^{2}
$$

i.e.,

$$
v_{i} v_{i j}=-\lambda(1+\varepsilon+a) v v_{j}, \quad 1 \leq j \leq n .
$$

Also at $P$

$$
\begin{aligned}
0 \geq & \frac{1}{2} \Delta G(P)=\sum_{i, j} v_{i j}^{2}+\nabla v \cdot \nabla(\Delta v)+\lambda(1+\varepsilon+a) v \Delta v+\lambda(1+\varepsilon+a)|\nabla v|^{2} \\
= & \sum_{i, j} v_{i j}^{2}-|\nabla v|^{2}-2 \nabla v \cdot \nabla\left(\nabla v \cdot \nabla \log f_{1}\right)-\lambda^{2}(1+\varepsilon+a) v(v+a) \\
& -2 \lambda(1+\varepsilon+a) v\left(\nabla v \cdot \nabla \log f_{1}\right)+\lambda(1+\varepsilon+a)|\nabla v|^{2} .
\end{aligned}
$$

If $\nabla v(P)=0$, then (12) is valid. If $\nabla v(P) \neq 0$, we can choose an orthonormal frame such that $v_{i}(P)=0,2 \leq i \leq n$, and $v_{1}(P) \neq 0$. (13) gives

$$
v_{11}=-\lambda(1+\varepsilon+a) v, \quad v_{1 i}=0, \quad 2 \leq i \leq n .
$$


Putting (15) into (14), we obtain

$$
\begin{aligned}
0 \geq & v_{11}^{2}+(\varepsilon+a) \lambda|\nabla v|^{2}-2 v_{1}\left(\nabla v \cdot \nabla \log f_{1}\right)_{1}-\lambda^{2}(1+\varepsilon+a) v^{2} \\
& -\lambda^{2}(1+\varepsilon+a) a v-2 \lambda(1+\varepsilon+a) v\left(\nabla v \cdot \nabla \log f_{1}\right) \\
= & \lambda^{2}(1+\varepsilon+a)^{2} v^{2}-\lambda^{2}(1+\varepsilon+a) v^{2}+(\varepsilon+a) \lambda|\nabla v|^{2}-\lambda^{2}(1+\varepsilon+a) a v \\
& -2 \sum_{i=1}^{n} v_{1} v_{i 1}\left(\log f_{1}\right)_{i}-2 \sum_{i=1}^{n} v_{1} v_{i}\left(\log f_{1}\right)_{i 1} \\
& -2 \lambda(1+\varepsilon+a) v v_{1}\left(\log f_{1}\right)_{1} \\
= & \lambda(\varepsilon+a)\left[|\nabla v|^{2}+\lambda(1+\varepsilon+a) v^{2}\right]-\lambda^{2}(1+\varepsilon+a) a v-2 v_{1} v_{11}\left(\log f_{1}\right)_{1} \\
& -2 v^{2}\left(\log f_{1}\right)_{11}-2(1+\varepsilon+a) v v_{1}\left(\log f_{1}\right)_{1} \\
= & \lambda(\varepsilon+a) G(P)-\lambda^{2}(1+\varepsilon+a) a v+2 v_{1} \lambda(1+\varepsilon+a) v\left(\log f_{1}\right)_{1} \\
& -2 v_{1}^{2}\left(\log f_{1}\right)_{11}-2 \lambda(1+\varepsilon+a) v v_{1}\left(\log f_{1}\right)_{1} .
\end{aligned}
$$

Hence

$$
0 \geq \lambda(\varepsilon+a) G(P)-\lambda^{2}(1+\varepsilon+a) a v-2 v_{1}^{2}\left(\log f_{1}\right)_{11} .
$$

Since $W$ and $\Omega$ are all convex, $\log f_{1}$ is concave $[\mathbf{1}]$, in particular, $-\left(\log f_{1}\right)_{11}(P) \geq$ 0 . Noting that $v \leq 1$, we have

$$
\lambda(1+\varepsilon+a) a \geq(\varepsilon+a) G(P), \quad G(x) \leq \lambda(1+\varepsilon+1) \frac{a}{\varepsilon+a} \leq \lambda(1+\varepsilon+a) .
$$

From (12) and (17) we can obtain that $G(x) \leq(1+\varepsilon+a) \lambda, x \in \bar{\Omega}$. This is

$$
|\nabla v|^{2} \leq \lambda(1+\varepsilon+a)\left(1-v^{2}\right) \leq \lambda(1+\varepsilon+a)\left(b^{2}-v^{2}\right) .
$$

Letting $\varepsilon \rightarrow 0$, we complete the proof of the lemma. Q.E.D.

LEMMA $3 . \lambda \geq 1 /(1+a) \cdot \pi^{2} / d^{2}$.

ProOF. By Lemma 2, we have

$$
\frac{|\nabla(v / b)|}{\sqrt{1-(v / d)^{2}}} \leq \lambda^{1 / 2}(1+a)^{1 / 2} .
$$

Suppose that $q_{1}$ and $q_{2} \in \bar{\Omega}$ such that $v\left(q_{1}\right)=1, v\left(q_{2}\right)=-1$, and let $L$ be the line segment between $q_{1}$ and $q_{2}$. L lies on $\bar{\Omega}$ completely, because it is convex. We integrate both sides of (18) along $L$ from $q_{2}$ to $q_{1}$ and obtain

$$
\lambda^{1 / 2}(1+a)^{1 / 2} d \geq \lambda^{1 / 2}(1+a)^{1 / 2} \text { length of } L \geq \arcsin \frac{1}{b}-\arcsin \frac{-1}{b} .
$$

For any $b>1,(19)$ is valid and, letting $b \rightarrow 1$, the lemma is completely proved. Q.E.D.

If $a=0$, then the Theorem is proved. Now suppose $a>0$. From Lemma 2

$$
\frac{|\nabla(v / b)|^{2}}{1-(v / b)^{2}} \leq \lambda(1+a), \quad b>1 .
$$

Set $\theta: \bar{\Omega} \rightarrow R, \theta=\arcsin (v / b), \arcsin (-1 / b) \leq \theta \leq \arcsin (1 / b)$. Then

$$
\frac{|\nabla(v / b)|^{2}}{1-(v / b)^{2}}=|\nabla \theta|^{2} \leq \lambda(1+a) \text {. }
$$


Obviously, $\nabla \theta=0$ if $v=0$. Define $F:[\arcsin -1 / b, \arcsin 1 / b] \rightarrow R$ as

$$
F\left(\theta_{0}\right)=\max _{\substack{x \in \bar{\Omega} \\ \theta(x)=\theta_{0}}} \frac{|\nabla(v / b)|^{2}}{1-(v / b)^{2}} .
$$

$F\left(\theta_{0}(x)\right)$ is continuous on $\bar{\Omega}$ and

$$
F\left(\theta_{0}\right) \leq \lambda(1+a), \quad \theta_{0} \in[\arcsin -1 / b, \arcsin 1 / b] .
$$

For any $\theta_{0} \in[\arcsin -1 / b, \arcsin 1 / b]$ there must be an $x_{0} \in \bar{\Omega}$ such that

$$
\theta\left(x_{0}\right)=\theta_{0} \quad \text { and } \quad F\left(\theta_{0}\right)=\frac{|\nabla(v / b)|^{2}}{1-(v / b)^{2}}\left(x_{0}\right)
$$

are valid. Since $a>0$, we can define a continuous function $\varphi$ on $\bar{\Omega}$ which satisfies

$$
F(\theta) \equiv \lambda\left(1+\frac{a}{b} \varphi(\theta)\right), \quad \varphi(\theta) \leq b .
$$

LEMMA 4. The $C^{\infty}$ function $y:[\arcsin -1 / b, \arcsin 1 / b] \rightarrow R$ satisfies

(i) $y(\theta) \geq \varphi(\theta), \theta \in[\arcsin -1 / b, \arcsin 1 / b]$;

(ii) there is an $x_{0} \in \bar{\Omega}$ such that $\theta\left(x_{0}\right)=\theta_{0}$ and $y\left(\theta_{0}\right)=\varphi\left(\theta_{0}\right)$;

(iii) $y(\theta) \geq-1$ for any $\theta \in[\arcsin -1 / b$, $\arcsin 1 / b]$;

(iv) $y^{\prime}\left(\theta_{0}\right) \geq 0$.

Then the following inequality is valid:

$$
\varphi\left(\theta_{0}\right) \leq \sin \theta_{0}-y^{\prime}\left(\theta_{0}\right) \sin \theta_{0} \cos \theta_{0}+\frac{1}{2} y^{\prime \prime}\left(\theta_{0}\right) \cos ^{2} \theta_{0} .
$$

ProOF. Consider the function $\Phi(x): \bar{\Omega} \rightarrow R$,

$$
\Phi(x)=\left\{\frac{|\nabla v|^{2}}{b^{2}-v^{2}}-\lambda(1+c y)\right\} \cos ^{2} \theta,
$$

where $b>1$ and $c=a / b$. Obviously, $\Phi(x) \leq 0$ for $x \in \bar{\Omega}$ and $\Phi\left(x_{0}\right)=0$, i.e., $\Phi(x)$ attains its maximum at $x_{0}$, since

$$
\Phi(x)=\frac{1}{b^{2}}|\nabla v|^{2}-\lambda\left(1-\frac{v^{2}}{b^{2}}\right)(1+c y) .
$$

If $\nabla v\left(x_{0}\right)=0$, then

$$
0=\Phi\left(x_{0}\right)=-\left.\lambda\left(1-v^{2} / b^{2}\right)(1+c y)\right|_{x_{0}}
$$

and

$$
y\left(x_{0}\right)=-1 / c=-a / b<-1 .
$$

This contradicts the assumption (iii). Thus $\nabla v\left(x_{0}\right) \neq 0$. By Lemma $1, x_{0} \notin \partial \Omega$, i.e., $x_{0} \in \Omega$. According to the maximum principle, we have

$$
\begin{aligned}
& \nabla \Phi\left(x_{0}\right)=0, \\
& \Delta \Phi\left(x_{0}\right) \leq 0 .
\end{aligned}
$$

For convenience we write $\Phi(x)$ as

$$
\Phi(x)=\frac{1}{b^{2}}|\nabla v|^{2}-\cos ^{2} \theta(1+c y) .
$$


Then

$$
\Phi_{j}=\frac{1}{b^{2}} \sum_{i} v_{i} v_{i j}-\lambda(1+c y)(-2 \cos \theta \sin \theta) \theta_{j}-c \lambda \cos ^{2} \theta y^{\prime} \theta_{j} .
$$

(22) gives that at $x_{0}$

$$
\frac{2}{b^{2}} \sum_{i} v_{i} v_{i j}=\lambda\left[c y^{\prime} \cos ^{2} \theta-2(1+c y) \cos \theta \sin \theta\right] \theta_{j}, \quad 1 \leq j \leq n .
$$

And also

$$
\begin{aligned}
\Delta \Phi= & \frac{2}{b^{2}} \sum_{i, j} v_{i j}^{2}+\frac{2}{b^{2}} \nabla v \cdot \nabla(\Delta v)-\lambda c \cos ^{2} \theta \Delta y \\
& -\lambda(1+c y) \Delta \cos ^{2} \theta-2 \lambda c \nabla \cos ^{2} \theta \cdot \nabla y \\
= & \frac{2}{b^{2}} \sum_{i, j} v_{i j}^{2}+\frac{2}{b^{2}} \nabla v \cdot \nabla(\Delta v)-\lambda c \cos ^{2} \theta\left(y^{\prime \prime}|\nabla \theta|^{2}+y^{\prime} \Delta \theta\right) \\
& +4 \lambda c y^{\prime} \cos \theta \sin \theta|\nabla \theta|^{2}-\lambda(1+c y) \Delta \cos ^{2} \theta .
\end{aligned}
$$

From (23) we have that at $x_{0}$

$$
\begin{aligned}
0 \geq & \frac{2}{b^{2}} \sum_{i, j} v_{i j}^{2}+\frac{2}{b^{2}} \nabla v \cdot \nabla(\Delta v)-\lambda c \cos ^{2} \theta\left(y^{\prime \prime}|\nabla \theta|^{2}+y^{\prime} \Delta \theta\right) \\
& +4 \lambda c y^{\prime} \cos \theta \sin \theta|\nabla \theta|^{2}-\lambda(1+c y) \Delta \cos ^{2} \theta
\end{aligned}
$$

Since $\nabla v\left(x_{0}\right) \neq 0$, we can choose an orthonormal frame such that $v_{1}\left(x_{0}\right) \neq 0$ and $v_{i}\left(x_{0}\right)=0,2 \leq i \leq n$. Then by $\left(22^{\prime}\right)\left(\right.$ note $\left.\sin \theta=v / b, \theta_{j}=v_{j} / b \cos \theta\right)$

$$
\begin{aligned}
& v_{i 1}=0, \quad 2 \leq i \leq n, \\
& v_{11}=(b / 2) \lambda\left[c y^{\prime} \cos \theta-2(1+c y) \sin \theta\right] .
\end{aligned}
$$

Now we compute the terms in $\left(23^{\prime}\right)$ at the particular frame

$$
\begin{aligned}
\nabla v \cdot \nabla(\Delta v)_{x_{0}} & =\sum_{i, j} v_{i} v_{j j i}=\sum_{j} v_{1}\left(v_{j j}\right)_{1} \\
& =v_{1}\left[-\lambda(v+a)-2 \sum_{i} v_{i}\left(\log f_{1}\right)_{i}\right]_{1} \\
& =-\lambda v_{1}^{2}-2 v_{1} \sum_{i} v_{i 1}\left(\log f_{1}\right)_{i}-2 v \sum_{i} v_{i}\left(\log f_{1}\right)_{i 1} \\
& =-\lambda v_{1}^{2}+2 v_{1} v_{11}\left(\log f_{1}\right)_{1}=2 v_{1}^{2}\left(\log f_{1}\right)_{11} \quad(\because(24)) .
\end{aligned}
$$

From $\Delta v / b=\Delta \sin \theta=\cos \theta \Delta \theta-\sin \theta|\nabla \theta|^{2}$, we have

$$
\Delta \theta=\frac{1}{\cos \theta}\left[\frac{\Delta v}{b}+\sin \theta|\nabla \theta|^{2}\right] \text {. }
$$

And

$$
\begin{aligned}
\Delta \cos \theta & =\Delta\left(1-\frac{v^{2}}{b^{2}}\right)=-\frac{1}{b^{2}} \Delta v^{2}=-\frac{2}{b^{2}}\left(v \Delta v+|\nabla V|^{2}\right) \\
& =-\frac{2}{b^{2}} v \Delta v-2 \cos ^{2} \theta|\nabla \theta|^{2}
\end{aligned}
$$


From $\Phi\left(x_{0}\right)=0$, we have at $x_{0}$

$$
|\nabla \theta|^{2}=\frac{1}{b^{2}} \frac{|\nabla v|^{2}}{\cos ^{2} \theta}=\frac{1}{b^{2}} \frac{|\nabla v|^{2}}{1-(v / b)^{2}}=\frac{|\nabla v|^{2}}{b^{2}-v^{2}}=\lambda(1+c y) .
$$

Putting (25)-(28) into $\left(23^{\prime}\right)$, we obtain that at $x_{0}$

$$
\begin{aligned}
0 \geq & \frac{2}{b^{2}} \sum_{i, j} v_{i j}^{2}+\frac{2}{b^{2}}\left[-\lambda v_{1}^{2}-2 v_{1} v_{11}\left(\log f_{1}\right)_{1}-2 v_{1}^{2}\left(\log f_{1}\right)_{11}\right] \\
& -\lambda c\left[\lambda y^{\prime \prime}(1+c y)+y^{\prime}\left(\frac{\Delta v}{b \cos \theta}+\frac{\sin \theta}{\cos \theta}\right) \lambda(1+c y)\right] \cos ^{2} \theta \\
& +4(1+c y) \lambda^{2} c y^{\prime} \cos \theta \sin \theta-\lambda(1+c y)\left[-\frac{2}{b^{2}} v \Delta v-2 \lambda \cos ^{2} \theta(1+c y)\right] .
\end{aligned}
$$

Since $|\nabla v|^{2}=v_{1}^{2}=b^{2} \cos ^{2} \theta|\nabla \theta|^{2}=\lambda^{2} \cos ^{2} \theta(1+c y)(\because(28))$ and $\Delta v=$ $-\lambda(v+a)-2 \nabla v \cdot \nabla \log f_{1},(29)$ can be written as

$$
\begin{aligned}
0 \geq \frac{2}{b^{2}} \sum_{i, j} v_{i j}^{2}-2 \lambda^{2}(1+c y) \cos ^{2} \theta-\lambda^{2} c y^{\prime \prime}(1+c y) \cos ^{2} \theta \\
\quad+2 \lambda^{2} c y^{\prime} \cos \theta(\sin \theta+c)+3 \lambda^{3} c y^{\prime}(1+c y) \sin \theta \cos \theta \\
\quad+2 \lambda(1+c y)^{2} \cos ^{2} \theta-2 \lambda^{2}(1+c y) \sin \theta(\sin \theta+c) \\
\quad-\frac{4}{b^{2}} v_{1}^{2}\left(\log f_{1}\right)_{11}-\frac{4}{b^{2}} v_{1} v_{11}\left(\log f_{1}\right)_{1} \\
\quad+\frac{2}{b} \lambda\left[c y^{\prime} \cos \theta-2(1+c y) \sin \theta\right] v_{1}\left(\log f_{1}\right)_{1} .
\end{aligned}
$$

Putting the second formula of (24) into the above inequality and noting that $\left(\log f_{1}\right)_{11} \leq 0$, we have

$$
\begin{aligned}
0 \geq & \frac{1}{2} \lambda^{2} c^{2}\left(y^{\prime}\right)^{2} \cos ^{2} \theta+2 \lambda^{2}(1+c y)^{2}-2 \lambda^{2}(1+c y) \cos ^{2} \theta \\
& +\lambda^{2} c y^{\prime}[(1+c y) \sin \theta \cos \theta+\cos \theta(\sin \theta+c)] \\
& -\lambda^{2} c(1+c y) y^{\prime \prime} \cos ^{2} \theta-2 \lambda^{2}(1+c y) \sin \theta(\sin \theta+c) .
\end{aligned}
$$

Dividing both sides of the above inequality by $\lambda^{2}(1+c y)>0$, we have

$$
\begin{aligned}
& 0 \geq y^{\prime}\left(\sin \theta \cos \theta+\cos \frac{\sin \theta+c}{1+c}\right)-y^{\prime \prime} \cos ^{2} \theta+\frac{2}{c}(1+c y)-\frac{2}{c}-2 \sin \theta, \\
& 2 y-2 \sin \theta \leq y^{\prime \prime} \cos ^{2} \theta-y^{\prime}\left(\sin \theta \cos \theta+\cos \theta \frac{\sin \theta+c}{1+c y}\right) .
\end{aligned}
$$

Since $-1 \leq y\left(\theta_{0}\right)=\varphi\left(\theta_{0}\right) \leq b$, thus,

$$
\left|y\left(\theta_{0}\right)\right| \leq b, \quad y\left(\theta_{0}\right) \sin \theta_{0} \leq\left|y\left(\theta_{0}\right)\right|\left|\sin \theta_{0}\right| \leq b v\left(\theta_{0}\right) / b \leq 1
$$

and

$$
c \geq c y \sin \theta, \quad c+\sin \theta \geq(1+c y) \sin \theta, \quad \frac{c+\sin \theta}{1+c y} \geq \sin \theta .
$$

Since $y^{\prime}\left(\theta_{0}\right) \geq 0$, we have

$$
\varphi\left(\theta_{0}\right)=y\left(\theta_{0}\right) \leq \sin \theta_{0}-y^{\prime}\left(\theta_{0}\right) \sin \theta_{0} \cos \theta_{0}+\frac{1}{2} y^{\prime \prime}\left(\theta_{0}\right) \cos ^{2} \theta_{0} . \quad \text { Q.E.D. }
$$


LEMma 5. Define a function $\psi:[-\pi / 2, \pi / 2] \rightarrow R$ as

$$
\left\{\begin{array}{l}
\psi(\theta)=\frac{(4 / \pi)(\theta+\cos \theta \sin \theta)-2 \sin \theta}{\cos ^{2} \theta}, \quad \theta \in(-\pi / 2, \pi / 2) \\
\psi(-\pi / 2)=-1, \quad \psi(\pi / 2)=1 .
\end{array}\right.
$$

Then $\psi$ is a $C^{\infty}$ function in $(-\pi / 2, \pi / 2)$ and is continuous on $[-\pi / 2, \pi / 2]$ and also $y=\psi(\theta)$ satisfies the following equation:

$$
y-\sin \theta+y^{\prime} \sin \theta \cos \theta-\frac{1}{2} y^{\prime \prime} \cos ^{2} \theta=0,
$$

and $y^{\prime}(\theta) \geq 0, \theta \in(-\pi / 2, \pi / 2)$.

PROOF. See reference $[4]$.

LEMMA 6. Let $\varphi(\theta)$ be the function defined by $(21)$. Then

$$
\varphi(\theta) \leq \psi(\theta), \quad \theta \in\left[\arcsin \frac{-1}{b}, \arcsin \frac{1}{b}\right],
$$

where $\psi(\theta)$ is defined by $(30)$.

PROOF. We will use the reduction to absurdity. If

$$
\sigma=\varphi\left(\theta_{0}\right)-\psi\left(\theta_{0}\right)=\max _{\theta}\{\varphi(\theta)-\psi(\theta)\}>0
$$

we could choose $\psi(\theta)+\sigma=\tilde{y}$ as $y$ in Lemma 4 . Therefore, $\varphi\left(\theta_{0}\right)=\tilde{y}\left(\theta_{0}\right)=\psi\left(\theta_{0}\right)+\sigma \leq \sin \theta_{0}-\psi^{\prime}\left(\theta_{0}\right) \sin \theta_{0} \cos \theta_{0}+\frac{1}{2} \psi^{\prime \prime}\left(\theta_{0}\right) \cos ^{2} \theta_{0}=\psi\left(\theta_{0}\right)$. This contradicts (32). Q.E.D.

PROOF OF THE THEOREM. By Lemma 6, we have

$$
|\nabla \theta|^{2}=\frac{|\nabla v|^{2}}{b^{2}-v^{2}} \leq \lambda\left(1+\frac{a}{b} \psi(\theta)\right)
$$

where $\psi(\theta)$ is the function defined by (30). Hence,

$$
\lambda^{1 / 2} \geq \frac{|\nabla \theta|}{\sqrt{1+(a / b) \psi(\theta)}} .
$$

Obviously,

$$
\psi(0)=0, \quad \psi(-\theta)=-\psi(\theta) .
$$

Integrating both sides of (33) as in Lemma 3 , we obtain

$$
\begin{aligned}
\lambda^{1 / 2} d & \geq \int_{\arcsin -1 / b}^{\arcsin 1 / b} \frac{d \theta}{\sqrt{1+(a / b) \psi(\theta)}} \\
& =\int_{0}^{\arcsin 1 / b}\left(\frac{1}{\sqrt{1+(a / b) \psi(\theta)}}+\frac{1}{\sqrt{1-(a / b) \psi(\theta)}}\right) d \theta .
\end{aligned}
$$

Since $| \pm(a / b) \psi(\theta)| \leq 1$,

$$
\frac{1}{\sqrt{1+(a / b) \psi(\theta)}}+\frac{1}{\sqrt{1-(a / b) \psi(\theta)}}=2\left[1+\sum_{p-1}^{\infty} \frac{1 \cdot 3 \cdots(4 p-1)}{2 \cdot 4 \cdots(4 p)}\left(\frac{a}{b}\right)^{2 p} \psi^{2 p}\right] \geq 2 \text {. }
$$

Thus

$$
\lambda^{1 / 2} d \geq 2 \arcsin \frac{1}{b}, \quad \lambda \geq \frac{4}{d^{2}}\left(\arcsin \frac{1}{b}\right)^{2} .
$$

Letting $b \rightarrow 1$, we obtain $\lambda \geq \pi^{2} / d^{2}$. Q.E.D. 


\section{REFERENCES}

1. H. Brascamp and E. Liep, On extensions of the Brunn-Minkowski and Prëkopa-Leindler theorems, including inequalities for log concave function, and with an application to diffusion equation, J. Funct. Anal. 22 (1976), 366-389.

2. R. Courant and D. Hilbert, Methods of mathematical physics, vol. I, Interscience, New York, 1953.

3. Bun Wong, Shing-Tung Yau and Stephen S.-T. Yau, An estimate of the first two eigenvalues in the Schrödinger operator (to appear).

4. Jia-Qing Zong and Hong-Zhang Yang, Estimates of the first eigenvalue of Laplace operator on compact Riemannian manifolds, Sci. Sinica Ser. A 9 (1983), 812-820. (Chinese)

School of Mathematics, The Institute for Advanced Study, Princeton, NEW JERSEY 08540

Current address: Institute of Applied Mathematics, Academia Sinica, Beijing, People's Republic of China 